\title{
Strabismus surgery complications
}

\author{
Namita Sharma ${ }^{1, *}$, Reena Sharma ${ }^{2}$, B. D Sharma ${ }^{3}$, Asma Khan ${ }^{4}$ \\ ${ }^{1}$ Senior Resident, ${ }^{2}$ Assistant Professor, ${ }^{3}$ Professor, ${ }^{4}$ Junior Resident, Dept. of Ophthalmology, Uttar Pradesh University of \\ Medical Sciences, Saifai, Uttar Pradesh, India \\ *Corresponding Author: \\ Email: namitasharmajnmc@gmail.com
}

\begin{abstract}
Serious complications during or after strabismus surgery are infrequent. This article gives a brief idea of the complications either local or systemic that can occur during or after squint surgery. Meticulous hemostasis is required at each step in the procedure, whichever conjunctival approach to the muscle is used. Particular caution is required with patients who have thin sclera (such as myopes), have a history of scleritis, previous scleral procedures. The surgeon must be aware of the detailed surgical anatomy of extraocular muscles (eg.location of contiguous vortex veins, especially during oblique muscle surgery).
\end{abstract}

Keywords: Extraocular, Muscles, Strabismus.

\section{Introduction}

As with any other surgical procedure, some patients undergoing squint surgery may have complications. Majority of these complications are innocuous. Serious complications may occur but are uncommon.

The complications of squint surgery can be classified as local ophthalmologic complications and systemic complications. We have mainly discussed the local complications in this article. The systemic complications are usually anaesthesia related and can be avoided by taking detailed history of any systemic disease or any familial condition.

\section{Local Ophthalmological Complications}

They can be further classified to per operative and post operative complications.

\section{Peroperative Complications}

Surgery on the Wrong Muscle: It can be because of wrong identification of eye that has to undergo surgery. It is also possible to pick up the wrong muscle due to inadequate surgical exposure per operatively. Commonly inferior oblique is confused with inferior rectus or lateral rectus. To avoid this, one should not go too posterior while hooking the lateral rectus muscle. The rotation of the globe should be avoided and checked for to prevent holding the wrong muscle. This problem is common in patients with craniofacial dysostoses where whole orbits are excyclorted.

Globe Perforation: The reported incidence of scleral perforation varies from $0.2 \%$ to $7.8 \% .^{1,2}$ Serious concern after scleral perforation is increased risk of retinal detachment and post op endophthalmitis. Whenever perforation occurs during surgery, the retina should be examined immediately. The cryotherapy should be used to seal the perforation immediately. The patient should then be followed up by doing regular retinal examination, and referred to retina specialist if needed. The use of spatulated and small calibre needles for securing rectus muscles to the sclera is believed to have reduced the risk and incidence of scleral perforation. $^{3}$

Haemorrhage: Significant haemorrhage is rare but can occur while operating on inferior oblique muscle where belly rather than tendon is cut. It can also be caused by injury to vortex veins during superior rectus or superior oblique surgery. Sometimes the scleral muscle stump hemorrhages after the muscle has been dis inserted. An intra conjunctival or intramuscular hematoma occasionally may develop. It is important to control bleeding before continuing the surgery, as organization of the clot and subsequent scarring will affect the action of the muscle. Thus, we may not be able to achieve the desired surgical outcome. Most haemorrhages, particularly from a capillary bed, respond well to brief pressure with a cellulose sponge that may be soaked with a drop or two of 1:10,000 epinephrine. ${ }^{4}$ Cautious use of gentle diathermy is recommended prior to operating the muscles.

Slipped and Lost Muscles: The term "slipped muscle" generally refers to a dis inserted rectus muscle which retracts posteriorly into its capsule in the days immediately after surgery. It is one of the most distressing complications during strabismus surgery. It must be suspected when a large overcorrection with limitation of movement suddenly develops after initial alignment. The cause is believed to be mistaken placement of sutures in the muscle capsule instead of the muscle or muscle tendon. Neural imaging is indispensable in locating the slipped muscle prior to attempts to retrieve and reattach it. ${ }^{4}$ Treatment involves identification of a slipped muscle intraoperatively, followed by placement of sutures into the muscle/tendon tissue posterior to the muscle capsule and advancement of the muscle anteriorly.

When the muscle is lost during the operation, the surgeon, above all, must remain calm. Under bright illumination (headlight or microscope, if necessary), with the help of additional assistants and optimal 
exposure with malleable retractors, the area in which the muscle loss is suspected should be gently explored by hand-overhand grasping of tenon's capsule, to which posterior fibres of the muscle are usually attached. ${ }^{4}$

The direction of exploration should occur in the direction of the muscle, rather than toward the posterior pole where the muscle will rarely be found and injury to the optic nerve is a risk. ${ }^{4}$ Irrigation of the operative field with balanced salt solution may cause the pink colour of the muscle to contrast with the whitish colour of Tenon's capsule and thus help identification of even a few remaining muscle fibres. These should be immediately secured with a suture. ${ }^{4}$ It is difficult to locate medial rectus muscle which has no other attachments unlike other rectus muscles having few fibres attached to oblique muscles.

\section{Post-Operative Complications}

Under Corrections and Over Corrections: It is due to insufficient or excessive surgery performed for the angle of squint. Other causes include surgeries performed on scarred muscles or slippage of muscle. These complications can be avoided by doing meticulous preoperative assessment and using adjustable sutures in at risk patients.

If the cause of under correction and overcorrection is slippage of muscle then immediate reoperation is required otherwise it is better to allow the eyes to settle down and wait until further assessment can be made.

Anterior Segment Ischemia: It is a more serious complication of strabismus surgery. It usually results from simultaneous surgeries on three or four rectus muscles which results in with the inevitable disruption of blood supply to the anterior segment from the anterior ciliary arteries. Several cases are on record in which the patients developed anterior segment ischemia after surgery on just two opposing rectus muscles. ${ }^{5-8}$

It presents with ocular pain, blurred vision within 24 hrs of surgery. Clinical signs include microcystic epithelial edema and marked thickening of the cornea. Prominent folds occur in Descemet's membrane, and nonpigmented keratitic precipitates and a mild cellular aqueous humour reaction are usually present.], A fixed and distorted pupil, and cataract formation are late sequelae of this complication. Severe functional impairment of the eye and even phthisis bulbi has been reported. ${ }^{9}$

Although fluorescein iris angiography appeared to be a promising method to determine when collateral circulation developed after muscle surgery, ${ }^{10-13}$ the "safe" interval after which the second procedure can be planned is unknown and subject to wide individual variations. The probable mechanism of redistribution of blood flow to the anterior segment after disinserting the muscles is via the long posterior ciliary arteries. Routine preoperative angiograms are not recommended. As a general rule, it is advised to wait for at least 6 months before operating the vertical recti, if horizontal recti have been operated.

Treatment consists of intensive systemic and topical administration of corticosteroids. However, there is no evidence that visual outcome or the speed of resolution is influenced by such treatment. ${ }^{14}$

Anterior Advancement of the Plica Semilunaris: Plica semilunaris is a crescent shaped old of bulbar conjunctiva located at the medial canthus lateral to caruncle. Anterior advancement of the plica semilunaris is a serious potential complication of squint surgery on the medial rectus muscle. It is sometimes difficult to identify the edges of the conjunctival incision at the completion of surgery. This typically occurs when the plica becomes edematous and is confused with the wound edges, advancing the plica anteriorly and suturing it to the limbus. This complication is most likely to occur in patients with thin sclera and after prolonged surgery. Prevention involves awareness of the potential for this complication to occur combined with meticulous identification of the edges of the conjunctival incision during wound closure. The edges of the conjunctival incision can be marked preoperatively using either a sterile methylene blue marking pen or by placement of suture tags on the corner of the limbal incision. ${ }^{4}$

Subconjunctival Inclusion Cysts: These inclusion cysts are thought to occur when nests of conjunctival epithelial cells migrate in the episcleral space during squint surgery. These cells later proliferate and develop into a subconjunctival epithelial inclusion cyst. These cysts tend to enlarge over time and treatment is usually warranted. Treatment is surgical excision. Thermal cautery has been recommended for small conjunctival inclusion cysts. ${ }^{15}$

Dellen: A "delle"' (plural dellen) is characterized as a shallow clearly defined excavation at the peripheral aspect of the cornea. It typically develops during the first week or second week after surgery as a result of localized evaporation and dehydration of the cornea. The base of a delle is typically hazy and dry. The reported incidence of a delle formation ranges between $0.3 \%$ and $22.45 \% .^{16}$ Delle usually responds well to a firm bandage applied to the eye for 24 to 48 hours. It can be prevented by smooth closure of the limbal wound and resection of excess conjunctiva to prevent tissue elevation near the limbus.

A scleral delle after strabismus surgery consist of a dark, translucent scleral patch that disappear after hydration and reappear on dehydration. Patients often report that they have developed a "black hole" in their eye, as the underlying dark uvea becomes visible through the thinned sclera. These lesions spontaneously resolve and comfort and resolution may be hastened by administration of topical ophthalmic lubricants. ${ }^{17}$

Diplopia: Diplopia often occurs when a patient with non paralytic squint undergoes extraocular muscle surgery and the position of the deviated eye is changed 
so that the fixated object may no longer fall into the area of the suppression scotoma. ${ }^{4}$ How long it persists depends on the ability of the patient to suppress or to ignore the second image. Since this ability decreases with age, constant diplopia is more common in adults. To assess this risk preoperatively and to give the patient a chance to experience diplopia, fully or nearly fully correct the deviation with prisms placed in a trial frame in front of the glasses. Even if diplopia can be elicited in this way, this does not prove that it will be present after surgery. Preoperative intramuscular botulinum injection in the tighter muscle can be used to elicit diplopia.

Pyogenic Granulomas: Pyogenic granulomas" have been reported to occur after approximately $1 \%$ of all conjunctival incisions. ${ }^{18}$ Histologically, these lesions are composed of mixed acute and chronic inflammatory cells with capillary proliferation in a lobular pattern. Pyogenic granulomas typically develop 3 to 4 weeks after surgery. These lesions are usually self-limiting and resolve over several weeks. The use of topical steroids may hasten resolution, though evidence to support the use of topical steroids is anecdotal.

\section{Systemic Complications}

Serious systemic complications most often are related to exacerbation of concurrent systemic disease. The surgeon must always search for information with respect to unusual reactions to an anaesthetic agent by members of the patient's family, since there are several genetic conditions, such as hepatic porphyria and suxamethonium sensitivity, that cause severe complications during and after anaesthesia. ${ }^{19}$

A less harmful complication is bradycardia because of vagal stimulation, which results from pulling on the muscles, especially the medial rectus muscle. This oculocardiac reflex is a transient phenomenon and the surgeon must immediately stop any operative manipulation of the eye. The cardiac rhythm is usually restored after the pull on the muscle is relaxed, but intravenous injection of atropine is usually given by the anaesthesiologist at this point. Prophylactic Injection of atropine is recommended in all patients who are to undergo muscle surgery. Electrocardiographic monitoring is important during all types of eye surgery to detect potentially dangerous cardiac rhythm disturbances. ${ }^{20}$

\section{Conclusion}

Under correction or overcorrection of squint, intra operative haemorrhage, slipped muscle and globe perforation are common complications that can occur with squint surgery. These complications can however be easily avoided by careful planning and intraoperative management. The lost rectus muscle and anterior segment ischaemia are serious ocular complications, which have to be dealt with utmost care.

\section{References}

1. Park K, Hong S, Chung W, Kim SS, Byeon SH, Seong GJ, Lee JB, Han SH. Inadvertent scleral perforation after strabismus surgery: incidence and association with refractive error. Can J Ophthalmol. 2008;43(6):669-672.

2. Dang Y, Racu C, Isenberg SJ. Scleral penetrations and perforations in strabismus surgery and associated risk factors. J AAPOS. 2004;8(4):325-331.

3. Hussein MA, Coats DK, Harris LD, Sanchez CR, Paysse EA. Ultrasound biomicroscopy (UBM) characteristics of scleral tunnels created with suture needles commonly used during strabismus surgery. Binocul Vis Strabismus Q. 2007;22(2):102-108.

4. Von Noorden GK, Campos EC. Binocular vision and Ocular Motility: Theory and Management of Strabismus. Missouri: Mosby;2002.

5. MacEwen CJ, Lee JP, Fells P. Aetiology and management of the 'detached' rectus muscle. $\mathrm{Br} J$ Ophthalmol. 1992;76(3):131-136.

6. Göçmen ES, Atalay Y, Evren Kemer Ö, Sarıkatipoğlu HY. Anterior Segment Ischemia after Strabismus Surgery. Turk J Ophthalmol. 2017;47(1):47-51.

7. Yip WW, Yu CB, Fan DS, Yick DW, Rao SK, Lam DS. Anterior segment ischemia after two-muscle surgery in a patient with radiation-treated nasopharyngeal carcinoma. J Pediatr Ophthalmol Strabismus. 2008;45(1):40-42.

8. Smith JM, Hwang RY, Siringo F, Quiroz-Mercado H, Oliver SC, Mandava N, Mathias MT, Olson JL. Concurrent endophthalmitis and anterior segment ischemia after strabismus surgery. Retin Cases Brief Rep. 2017;11(2):148-151.

9. Vijayalakshmi P, Muralidhar R, Shetty S, Sane M. Resolution of anterior segment ischemia after the removal of lateral fixation sutures. J AAPOS. 2008;12(5):531-532.

10. Helveston EM: Muscle transposition procedures. Surv Ophthalmol. 16:92,1971.

11. Oltra EZ, Pineles SL, Demer JL, Quan AV, Velez FG. The effect of rectus muscle recession, resection and plication on anterior segment circulation in humans. $\mathrm{Br} \mathrm{J}$ Ophthalmol. 2015;99(4):556-560.

12. Olver JM, Lee JP. The effects of strabismus surgery on anterior segment circulation. Eye (Lond). 1989;3(Pt 3):318-326.

13. Olver JM, Lee JP. Recovery of anterior segment circulation after strabismus surgery in adult patients. Ophthalmology. 1992;99(3):305-315.

14. Rosenbaum AL, Santiago AP, eds: Clinical Strabismus Management. Principles and Surgical Management. Philadelphia, WB Saunders, 1999

15. Coats DK, Olitsky SE. Strabismus Surgery and its Complications. Berlin Heidelberg: Springer-Verlag; 2007.

16. Saunders RA, Bluestein EC, Wilson ME, Berland JE. Anterior segment ischemia after strabismus surgery. Surv Ophthalmol. 1994;38(5):456-466.

17. Hawkins AS, Hamming NA. Thermal cautery as a treatment for conjunctival inclusion cyst after strabismus surgery. J AAPOS. 2001;5(1):48-49.

18. Mai G, Yang S. Relationship between corneal dellen and tearfilm breakup time. Yan Ke Хие Bao. 1991;7(1):43-46.

19. Lin SB, Li ZY, Zhan XK, Cai JH. A case of corneoscleral dellen after medial rectus recession combined with pterygium resection in right eye. Eye Sci. 2012;27(4):198-201.

20. Espinoza GM, Lueder GT. Conjunctival pyogenic granulomas after strabismus surgery. Ophthalmology. 2005;112(7):1283-1286. 
21. Zsigmond EK, Starkweather WH, Duboff GS, Flynn KA. Abnormal creatine-phosphokinase isoenzyme pattern in families with malignant hyperpyrexia. Anesth Analg. 1972;51(5):827-840.

22. McGoldrich KE: Anesthesia and the eye. In Barash PG, Cullen BR, Stoelging RK, eds: Clinical Anesthesia. Philadelphia, JB Lippincott, 1989: 1055.

How to cite this article: Sharma N, Sharma R, Sharma BD, Khan A. Strabismus surgery complications. Ind J Clin Exp Ophthalmol. 2018;4(3):300-303. 